\title{
THE DEEPER AND THE RICHER MEANINGS OF MATHEMATICAL TEACHING IN ELEMENTARY SCHOOLS. II
}

GEORGE W. MYERS

College of Education

GOOD ARITHMETIC TEACHING IS A TRAINING IN CHOICE

I have said that many ways of doing things in arithmetic should be taught; because there is a widespread disposition among teachers to talk and teach as though there were only one way to do an arithmetical problem, and that all that needs be done is to learn this one way. The old-time notion that there is one method for addition, one for subtraction, etc., was the foster-parent of the method-haggling, and device-hunting that so dominated and mechanized arithmetic work in the recent past. But this is the day of the open-door policy in the matter of method and device. They are now regarded only as means to the great end of thinking. Some teachers can render the highest service through one type of method, others become more effective through another type. As with teachers so with pupils. Some pupils will more readily grasp the underlying thought through one type of presentation, and others through another type. The good teacher must have at his finger-tips many ways of exhibiting the truth being taught, and must have great resourcefulness and tact in their use. There must be more openmindedness, more mental springiness, more flexibility in the use of method and device in the schoolroom. The essential question with all method is: to what extent does it deepen interest and clarify, intensify, and facilitate thought?

As just suggested, different methods will meet this crucial question with varying degrees of success with different pupils. Pupils must therefore be given latitude of choice as to their methods of attacking and of working problems.

But choosing, to be effective, must be rationalized and liberated by a knowledge of the reasons on which the choice is made, 
and guided and steadied by suitable standards for estimating the relative merits of choices. One all-embracing standard of judgment for choosing is the principle of ease of action. This is always available for elementary-school mathematics. It throws the choice sometimes in favor of this method, and sometimes in favor of that. If the textbook has been properly written it will not fix upon a single method for each process, then carefully manufacture problems to exemplify this method to the exclusion of all others. With good teaching, problems are selected to exhibit conditions under which this or that method is the more convenient, the more advantageous; and care will always be taken that these problems shall cover fairly well the ground of the situations that arise most frequently in common affairs. The pedagogic aim must ever be so to open up and exhibit the thinking of an arithmetical topic, that its thought-values shall spread out into other than pure arithmetical fields. This is what we call the culture-value of mathematics.

Choice must also be liberated. The pupil who knows only one way suffers constraint in choosing. He necessarily takes the only route that promises a way out. In strictness he does not choose; because he cannot. He either blindly follows, or is driven. This is the necessary outcome of one-method teaching. To prevent this driving, sometimes miscalled teaching, teachers must qualify masterfully to teach pupils to choose from among different available methods of attacking and of solving problems; to choose the order of processes used in a given problem; to choose the best way of executing the several processes, and to some extent to choose problem-material. Then will arithmetic become a powerful factor in training for rational choosing in life's emergencies, and strong willing inevitably follows right choosing. Good conduct, good morals, are simply the sum-total of correct choices. Verily, good arithmetic teaching conduces to good character and right conduct.

To specify concrete opportunities, the following enumeration of alternatives of choice will serve to suggest the scope of available materials for choice:

Sums to be obtained by adding ( I) upward; (2) downward; 
(3) horizontally; (4) columns by parts, then the sums of the parts; (5) footing tables vertically and horizontally; (6) horizontally and vertically; $(7)$ by grouping into Io's, 2o's, etc.

Differences to be found (I) the usual way; (2) by making-up method; (3) with subtrahend over minuend; (4) subtrahend beside minuend; (5) increasing the subtrahend digit; (6) subtracting from left to right.

Products to be found (I) the usual way; (2) left-to-right multiplication; (3) by factors of multiplier; (4) by parts of multiplier, as $25 \times 36=20 \times 36+5 \times 36=30 \times 25+6 \times 25$, etc.; (5) by aliquot parts of IOO, I,000, etc.

Quotients to be found by dividing (I) the usual way; (2) quotient over dividend; (3) by factors of divisor; (4) by aliquot parts; (5) taking common factors out of dividend and divisor.

\section{COMMON FRACTIONS}

Fractions should be added and substracted-

I. By measurement exercises with common standards.

2. By divided lines, squares, oblongs, circles, etc.

3. By brisk formal exercises such as-

a) How many fourths in $1 / 2$ ? In $2 / 2$ ?

b) How many fourths in $\mathrm{I} / 2$ and $\mathrm{I} / 4$ ? In $\mathrm{I} / 2+2 / 4$ ? In $\mathrm{I} / 2$ $+3 / 4$ ?

c) How many fourths in $I / 2$ less $I / 4$ ? In $I / 2+2 / 4$ ? In $I / 2$ $+3 / 4$ ?

d) How many sixths in $1 / 3$ ? $2 / 3$ ? $3 / 3$ ?

e) How many sixths in $\mathrm{I} / 3+\mathrm{I} / 6$ ? In $\mathrm{I} / 3+2 / 6$ ? In $2 / 3+\mathrm{I} / 6$ ? In $2 / 3+2 / 6$ ?

f) How many sixths in $1 / 3-1 / 6$ ? In $2 / 3-1 / 6$ ? In $2 / 3-2 / 6$ ? In $5 / 6-1 / 3$ ?

4. By the customary formal procedure.

Fractions should be multiplied-

I. By use of common standards of distance, area, bulk, capacity, weight, time, etc.

2: By divided lines, squares, oblongs, circles, etc.

3. By the customary formal procedure, first using "of" for times symbol, and later, using "times." (Distinction of partition from division is strongly advised against.) 
4. Check multiplications by reversing steps.

Fractions should be divided-

I. By measurement with common standards.

2. By aid of dividing lines, squares, oblongs, circles, etc.

3. By getting common denominators, and comparing numerators.

4. By the process of inverting divisor and multiplying, rationally presented.

5. Check division by multiplying.

Note.-Multiplications and divisions of fractions must be as systematically and persistently checked as are the processes with whole numbers. Cancellation is of great service in this checking.

\section{DECIMAL FRACTIONS}

The meaning of a decimal fraction should be taught by relating it-

I. To whole numbers, as with monetary values, or with metric units.

2. To common fractions, as $1 / 4=$ how many hundredths? etc.

3. To the system of decimal notation for integers.

After showing that the point is only a means of "pointing out" which digit, or figure denotes ones, or units, all that is needed for decimal addition and subtraction is to proceed precisely as with whole numbers, and to point the sum or difference so that the points all stand in a vertical line.

In multiplication of decimals, the pointing is a little more difficult. Here the point should be located-

I. By writing easy decimals in common fraction form, and multiplying, then, writing the product in the decimal form.

2. By multiplying as with integers, and then, by shifting the point on the principle that $\frac{1}{10}, \frac{1}{100}$, etc., of multiplicand or multiplier must give $\frac{1}{10}, \frac{1}{100}$, etc., of the product.

3. By the customary mechanical procedure, checking by deciding roughly about what the result must be.

In division, the quotient should be pointed by dividing through as with whole numbers and then-

I. Marking off in some way as many places in the dividend as there are decimal figures in the divisor, and noting that the 
quotient figures are figures of a whole number until this place is reached.

$$
\text { 23.5) } \frac{8.164}{203.04}
$$

2. Shifting the point over the same number of figures in both dividend and divisor until there is but one figure to the left of the point in the divisor, and then ask, About what is $1 / 2,1 / 3,1 / 4$, etc., of the modified dividend, pointing the quotient number so as to make it as near this estimated quotient as possible, by pointing alone.

$$
\begin{gathered}
\text { EXAMPLE } \\
203.04 \text { divided by } 2.3 .5=\text { ? } \\
2.35) \frac{8.64}{20.304}
\end{gathered}
$$

3. The customary procedure, checking the pointing by estimating roughly about what the result must be.

If time permitted I would indicate for percentage and interest and mensuration similar alternatives of procedure which should be made available to grade pupils. But a word to the wise is sufficient.

\section{SPEED AND ACCURACY}

Many teachers who glibly profess themselves seeking to secure "speed and accuracy in figuring" are unfortunately not succeeding in securing either, if we may credit recent findings of both local and foreign observers. If the animating idea of teachers of arithmetic could be ascertained, I dare say that in most cases, it would be found that the thing they are actually striving to develop, instead of accuracy, is only a sort of technical deftness, or mechanical precision as to the externals of process ; and, instead of speed, the thing actually produced is nervousness, feverishness, and fidgetiness. So faded, so hackneyed has the phrase "speed and accuracy" become through canting speech and canting acts, that the terms "speed" and "accuracy" need reinforming with new life and meaning. The good old wine very much needs new bottles.

I wish to attempt a closer specification than is prevalent of the meaning of these terms for arithmetic teaching. Definitions, of 
course, do not necessarily help classroom practice. Only such definitions influence practice as become organic to the thoughtprocess of the practitioner. Definitions are, therefore, merely conditions precedent-not conditions sufficient-to good practice.

\section{WHAT IS ACCURACY?}

In the first place, accuracy in arithmetic is a matter of the mind. An act of thought is its sine qua non. No conception of accuracy can be generated in the learner, nor can the habit of accuracy be fostered, without thinking on the learner's part. One remembers or he does not remember. "He remembers inaccurately" is a contradiction, and "He remembers accurately" is tautology. There are no gradations of remembering. This is particularly true of formal number facts. They may, perhaps, be remembered perfectly or imperfectly; but not accurately or inaccurately. Pure memorizing touches accuracy vere remotely. On the contrary, there are degrees of accuracy and of inaccuracy. But the type of accuracy that has meaning for arithmetic involves the judgment.

In the next place, judgments are based on comparisons, and comparing judgments is thinking. Arithmetical accuracy involves two elements, one external and the other internal. The internal element is an idea, mental operation, or a judgment. The external element is its expression, usually in the form of a symbol or of a mechanical process. The two factors that are always present, always an organic part of arithmetical accuracy, are an idea and its expression. If either factor be lacking, all talk of accuracy is empty and misleading. The city superintendent-not in Kansaswho said recently: "We use all diligence to make our children quick and accurate in fundamental conceptions as pure memory work," must have forgotten these facts. An examination of his school the other day showed his children to be unusually deficient in both respects. Drill-masters who are striving to teach children to be quick and accurate through pure memory work, or through the agency of pure drill on the formal side of arithmetic always have failed, are now failing, and must ever fail for the reason that they are trying to make bricks without straw, and 
(more's the pity!) when there is available an abundance of straw. The thought-side of the work is the indispensable, inescapable, and indisputable condition to accuracy. It is clear that I am not here speaking of mechanical precision, which is quite another matter, and has its rightful place in arithmetic.

A definition of accuracy that will both work and serve as a guide in the schoolroom may be ventured at this point. Accuracy in school arithmetic, means adequacy of expression to idea.

All teaching that makes the pupil's ideal merely to get set results, even a large score of them, that shifts his thought from correct answers solely as a check on correct work, at once degrades his sense of accuracy, demoralizes his habits, and confuses his ideals of accuracy. Kansas teachers are not accused of such teaching; but are herewith simply warned against it.

The true conception of accuracy for arithmetic emerges for both teacher and pupil through measuring and the use of measurements. This is indeed one of the strong reasons that arithmetic work should be based on measuring, rather than on counting, ratioing, or "on the inducing of judgments of relative magnitudes," as some would have it. In measuring, the question at once arises, and remains up contimually, "With what degree of closeness should measurements be made?" This question will be answered by the teacher from two points of view, and by the pupil from only one. The teacher must ascertain the smallest unit of which the pupil has, or can readily form, a working conception, and he will not attempt to require the learner to work with a much smaller unit. This is the natural limit from the educational point of view. To find it, and to keep within it, is the business of the teacher.

But the nature of the problem must guide both teacher and pupil as to the degree of practical refinement to be sought. It is in the problem that the pupil's interest focuses, not in the way of it, nor in the educational meaning of it. If the problem be to find the number of plants for his flowerbed, it is unmeaning, even foolish, to carry results to fractions. If the problem mean the cutting of a member for a playhouse, the pupil knows, quite as well as the teacher, that an eighth or a sixteenth of an inch becomes 
a matter serious enough to be attended to. The problem need not call for the actual cutting, save in imagination. The pupil readily pictures to himself, from the more or less indirect suggestions of the arithmetical problem, what would be required, were he to attempt to do the actual cutting and measuring. With this measuring and cutting which he does in imagination he does not willingly take unwarranted liberties and violate metrical proprieties.

\section{SPEED DIFFERENT WITH DIFFERENT PUPILS}

A little attention to the spontaneous activities of children, when spontaneity is unchecked by adults, readily reveals that each child has a certain norm of speed of motor discharge. Careful observing of children at work shows an analogous norm of speed of mental activity. The child's normal mental gait is as thoroughly and characteristically a part of him as is his physical gait. At this gait he dispatches work most economically and most comfortably, and the output of his effort maintains a uniformly high quality and quantity. To this pitch the music of his soul is attuned. To this chord the rhythm of his nature most fully responds.

At this gait the child is able to maintain a steady and high potential of thought-concentration. To force him by external stimuli to work at a higher rate than his norm makes his thinking uncertain, jerky, and flighty, and soon dissipates his fund of mental energy. To permit him to work below his norm is to make his thinking wobbly, and ambling, and his attention scattering. His thought-movements resemble those of the dying top, or bicycle. Low potential activity encourages mental loafing, habituates the child to going about his tasks in a half-hearted way, and tends to satisfy him with half-achievement. It engenders and fosters effort that is below the highest levels of possibility. Thus it occurs that allowing children to work below their norm of speed and forcing them by extraneous means to go beyond it end in the same deplorable issue-dissipation, rather than concentration, of thought.

The arithmetic teacher must then study his pupils individually 
to ascertain as nearly as possible the norm of speed for each. $\mathrm{He}$ must seek to hold the effort of the pupil to the level of this norm. He should be neither dragged, coaxed, nor spurred beyond this rate for any great length of time, if at all. The pupil will raise his norm by working within it and close up to it, but not beyond it: Dullness, stupidity, and something like mental flabbiness, resulting in enfeebled powers of concentration, are the outcome of abnormally low-potential activity; while fitful and nervous activity, issuing in a sort of hysteria, standing also for enfeebled concentration, are the end of abnormally high-potential activity. Thus do overstrained and understrained effort miss the mark of educational efficiency on the same side. I suggest that it is dispatch, which means a reasonably high rate of turning off work of a uniformly high grade of excellence with comfort to the workman, that arithmetic teaching should strive for, rather than for mere speed.

\section{DRILL-WORK IN ARITHMETIC}

There is also much canting speech and more canting action about drill-work in elementary-school mathematics. Time can be taken here only to indicate the pedagogical place of drill in the learning process. Teachers easily habituate themselves to dealing with the problems of education only from the view-point of the teaching process. We have scores of volumes that deal with the teaching process to one that deals explicitly with the learning process. Learning, with children as with adults, means acquiring, assimilating, and getting working control over new ideas and over processes for dealing with them. For purposes of a better understanding of it, the learning process may well be thought of as made up of three stages: viz., (I) the informal-use stage; (2) the formal-study stage; and (3) the application stage. Each of these may easily be subdivided into smaller steps as is exhibited below.

The formal-study stage of the learning process is made up of the following three pretty clearly marked steps: (I) the graphic step, in which the idea or process being learned is made to stand out before the learner in some pictured way. This is 
the psychological place for geometrized arithmetic; (2) the memory-step, in which the learner makes an explicit effort to remember the formal aspect of the object of study. This is the point at which generalized, or algebraized, arithmetic is most helpful; (3) the reflex-action step, in which the learner seeks to acquire automatic control of the new possession, to the end that the mechanics of it may no longer retard, but may facilitate, thinking. This is the psychologic moment and place for drill and it belongs nowhere earlier than this in the learning process; (4) the last stage of the learning process is the application stage. The learner here uses the newly acquired possession; but now he has already made it fully his own. He uses it with design, of destined purpose, and that he may feel how much he has added to his efficiency by having mastered it. $\mathrm{He}$ uses it for a time alone, and then among other familiar ideas, that he may both know it as an idea and as it appears in the midst of its natural meanings and settings.

To summarize: the learning process consists of the following stages and steps :

$$
\begin{gathered}
\text { The learning } \\
\text { process }
\end{gathered}\left\{\begin{aligned}
& \text { II. The informal-use stage. }\left\{\begin{array}{l}
\text { I. Occasional uses. } \\
\text { 2. Collated uses. }
\end{array}\right. \\
& \text { III. The formal-study stage }\left\{\begin{array}{l}
\text { I. The graphic step. } \\
\text { 2. The memory step. } \\
\text { 3. The reflex-action step (drill). }
\end{array}\right. \\
& \text { III. Use of the idea alone. } \\
& \text { 2. Use of the idea among others. }
\end{aligned}\right.
$$

For the pupil brisk, snappy, and properly timed drill-exercises, which never should be too long sustained, perform the following important educational offices:

I. Strengthen the power of voluntary attention, or of concentration of thought.

2. Develop facility of thought and ease of action.

3. Beget and foster a sense of growth in efficiency.

4. Cultivate quickness and sprightliness of memory.

5. Give readiness, alertness, and mechanical precision.

6. Show the need and value of knowledge that is available at the instant when, and at the spot where, it is wanted; i. e., of the "spot-cash" type of knowledge. 


\section{SUMMARY}

I may now summarize most of the points I have tried to make as follows :

I. The teacher may aid independence of thought in arithmetic in the following ways:

a) By accrediting the pupil's mode of thought;

b) By habitual checking of work;

c) By emphasizing the thought side of the work;

d) By impressing the thought that in arithmetic nothing is to be taken on authority;

e) By employing many semi-original problems.

2. Teachers may make arithmetic teaching a training in choice-

a) By having pupils do things in more than one way, to find the better way;

b) By showing reasons for standard conventions;

c) By allowing pupils to use their own way until the better way appeals to them as being the better way;

d) By allowing pupils to take the consequences of their own choosing of ways;

$e$ ) By using good ways themselves and by commending good ways of working when they are used by pupils.

3. Arithmetic may be made a training in judgment-

a) By teaching rational calculating;

b) By much antecedent estimating, followed immediately by measuring and calculating;

c) By having pupils to think through lists of exercises, or problems, without figuring, and to tell definitely just how they would solve them; in cases of doubt follow with the actual solutions ;

d) By having pupils always on the alert to find the easy way, even where the teacher feels quite sure his way is the easy way;

$e)$ By asking, after solutions, always in cases of doubt, whether the result seems about what it should be;

f) By independent checking.

4. Arithmetic work may be made a valuable training in concentration of thought- 
a) By brisk, snappy, drill-exercises, not too long sustained;

b) By having pupils work problems with a view to seeing how soon they can solve them correctly, the demand for speed being as natural as possible;

c) By having pupils see how many exercises they can get right in 5 , Io, or 20 minutes;

d) By estimating about what results must be;

$e$ ) By postponing technique and putting drill-work in its proper place in the teaching process;

f) By working always close up to, but not beyond, the pupil's norm of speed;

g) By mental calculating, counting by 2's, 3's, 5's, IO's, 4's, etc.

5. To make arithmetic work give training in clear thinking and in reasonable accuracy-

a) Give time enough, not too much, for difficult problems to be worked and checked;

b) By diagramming and sketching of figures (pictures) to advance the pupil's power to image number-relations;

c) By doing less "talky-talking" and more chalk-talking, and by the use of cross-lined paper and blackboard;

d) By recognizing in practice that attainable accuracy is different in different grades;

$e$ ) By studying habits of thinking of pupils, and insisting always on attainable neatness and system;

f) By using problem-material in which the pupil has, or may develop, an interest.

6. Arithmetic work may be made valuable training in inference-making-

a) By much preliminary estimating;

b) By careful estimating, before figuring, about what the result must be;

c) By much use of cancellation;

d) By much citation of reasons, not rules, for steps taken;

$e$ ) By rudimentary algebraic thinking, as $3 x$ is 12 , what is $x$ ? $3+\mathrm{x}$ is 8 , what is $x$ ? etc.

f) By the use of indirect measurements, as to get the side, 
or angle, of a triangle that cannot be measured directly but must be calculated from its relation to lines, or angles, that can be measured;

g) By asking why the result, as G.C.D., or L.C.M., etc., is what it claims to be ;

h) By set exercises in inference-drawing.

I believe your adopted texts will, so soon as teachers get into their spirit and method, go far toward securing these very real, very useful, but too-long neglected, virtues of good arithmetic teaching. We are laying no specious claim to making the arithmetic easy; but we do claim not to be making it hard. Our problem is the improvement of arithmetical opportunities now running too largely to waste. The inclusion of the deeper and richer educational meanings of arithmetic among teaching aims necessarily adds something to the teacher's responsibility. But where is the teacher, worthy the name, who will not say at once that the slight additional burden-for it is but slight-is not eminently worth while, in view of the immense importance of the issues of educating children?

\section{CONCLUSION}

In conclusion, let me say modernizing the teaching and the substance of elementary-school mathematics is mainly a problem of enlarging the scope of pedagogic view, and of making methodology more natural and more childlike than has been the wont. This can be done by, and only by, giving teachers a larger, a truer, a less static, a more dynamic conception of their professional duties touching this particular subject. Teachers must believe in arithmetic as an educational agency, before they can succeed with it educationally. Great indeed is the need for a profounder pedagogic faith in the sentiment-

I doubt not through the ages one increasing purpose runs,

And the thoughts of men are broadened with the process of the sunseven for the arithmetic. Then mellowed by that sweet docility of spirit that finds its sublimest expression in, "a little child shall lead," the teacher may go forth to meet the problems of improve- 
ment with courage, shod with sandals of wisdom and gentleness, that knows not the words failure, defeat, and discouragement. May the larger view, the more childlike view, the more hopeful spirit, and the forward look, be the inspiring watchword and portion of us all in the labor of reconstructing the good that now is into the better that is to be! 